\title{
The Internal Recycle Reactor Enhances Porous Calcium Silicate Hydrates to Recover Phosphorus from Aqueous Solutions
}

\author{
Wei Guan ${ }^{1}$ and Shichao Tian ${ }^{2}$ \\ ${ }^{1}$ Chongqing Key Laboratory of Environmental Materials \& Remediation Technologies, Chongqing University of Arts and Sciences, \\ Chongqing 402160, China \\ ${ }^{2}$ Shenzhen Environmental Science and New Energy Technology Engineering Laboratory, Tsinghua-Berkeley Shenzhen Institute, \\ Shenzhen 518055, China \\ Correspondence should be addressed to Shichao Tian; 18812672619@163.com
}

Received 31 May 2017; Accepted 27 August 2017; Published 26 October 2017

Academic Editor: Nicolas Roche

Copyright ( 2017 Wei Guan and Shichao Tian. This is an open access article distributed under the Creative Commons Attribution License, which permits unrestricted use, distribution, and reproduction in any medium, provided the original work is properly cited.

In this experiment, the porous calcium silicate hydrates (P-CSHs) were prepared via a hydrothermal method and then modified by polyethylene glycol (PEG). The modified P-CSHs combined with an internal recycle reactor could successfully recover the phosphorus from electroplating wastewater. The modified P-CSHs were characterized by X-ray diffraction (XRD), $\mathrm{N}_{2}$ adsorptiondesorption isotherms, and Fourier transform infrared spectroscopy (FT-IR). After compared with different samples, the modified P-CSHs-PEG2000 sample had larger specific surface area of $87.48 \mathrm{~m}^{2} / \mathrm{g}$ and higher pore volume of $0.33 \mathrm{~cm}^{3} / \mathrm{g}$, indicating a high capacity for phosphorus recovery. In the process of phosphorus recovery, the $\mathrm{pH}$ value of solution was increased to 9.5 , which would enhance the recovery efficiency of phosphorus. The dissolution rate of $\mathrm{Ca}^{2+}$ from P-CSH-PEG2000 was fast, which was favorable for phosphorus precipitation and phosphorus recovery. The effects of initial concentration of phosphorus, P-CSHs-PEG2000 dosage, and stirring speed on phosphorus recovery were analyzed, so the optimal operation conditions for phosphorus recovery were obtained. The deposition was analyzed by XRD, $\mathrm{N}_{2}$ adsorption-desorption, and SEM techniques; it was indicated that the pore volume and surface area of the P-CSHs-PEG2000 were significantly reduced, and the deposition on the surface of P-CSHs-PEG2000 was hydroxyapatite.

\section{Introduction}

In recent years, the electroplating industry is developing rapidly and the yield of electroplating wastewater is up to 4 billion $\mathrm{m}^{3}$ each year [1]. Cyanide has been gradually replaced by phosphate in plating cleaning, descaling, and anticorrosion due to the lower toxicity of phosphate [2]. The total concentration of phosphorus in electroplating wastewater is higher than $200 \mathrm{mg} / \mathrm{L}$. In the process of electroplating wastewater treatment, people focus on the removal of heavy metals and toxic anion and ignore the phosphorus resource recycling, causing huge loss of phosphorus resources. However, natural reserves of high-grade rock phosphorus are limited and dissipated on a global scale [3, 4]. Hence, considerable attention has been paid to phosphorus recovery for realizing closed-loop phosphorus recycling [5].
In recent years, various methods have been investigated to remove or recover phosphorus from wastewater, such as chemical precipitation [6], adsorption $[7,8]$, ion exchange [9], electrocoagulation [10], and membrane filtration [11]. Among these methods, chemical precipitation is difficult to be used to treat high concentration of phosphorus. In some areas, electrocoagulation and membrane filtration cannot be adopted due to the higher energy cost $[12,13]$. Adsorption process is widely used for the recovery of phosphorus. However, with respect to the sustainable recycling of phosphorus, adsorption techniques are limited owing to the difficult regeneration of adsorbents and subsequent complex desorption process [14]. Therefore, it is urgent to develop a simple and beneficial method for phosphorus recovery.

Compared with other adsorbents, calcium silicate hydrates (CSHs) show unique characteristics for phosphorus 
TABLE 1: Chemical components of carbide residue and white carbon black ((1) carbide residue; (2) white carbon black).

\begin{tabular}{|c|c|c|c|c|c|c|c|c|c|c|}
\hline & \multicolumn{10}{|c|}{ Chemical composition/\% } \\
\hline & $\mathrm{CaO}$ & $\mathrm{SiO}_{2}$ & $\mathrm{Al}_{2} \mathrm{O}_{3}$ & $\mathrm{SO}_{2}$ & $\mathrm{MgO}$ & $\mathrm{Fe}_{2} \mathrm{O}_{3}$ & $\mathrm{SrO}$ & $\mathrm{NaOH}$ & $\mathrm{CuO}$ & $\mathrm{H}_{2} \mathrm{O}$ \\
\hline (1) & 79.34 & 3.57 & 2.14 & 1.22 & 0.62 & 0.21 & 0.26 & - & - & 12.64 \\
\hline (2) & 0.08 & 97.46 & 0.16 & 1.82 & - & 0.03 & - & 0.29 & 0.02 & 0.14 \\
\hline
\end{tabular}

recovery. Firstly, the higher phosphorus adsorption capacity of CSHs is $137 \mathrm{mg} / \mathrm{g}$ [15], while the maximum adsorption capacity of phosphorus for natural calcium-rich sepiolite is $34 \mathrm{mg} / \mathrm{g}$ [16] and for ferrihydrite-modified diatomite it is $37 \mathrm{mg} / \mathrm{g}$ [17]. Secondly, CSHs are inexpensive and easily available materials compared with ferrihydrite-modified diatomite, lanthanum-modified bentonite clay, and zirconiumbased oxides [18]. Thirdly, CSHs are nontoxic inorganic material made of carbide residue and white carbon black by hydrothermal synthesis method [19]. Therefore, CSHs are kinds of ideal materials for phosphorus recovery.

The internal recycle reactor for phosphorus recovery is designed with both inner and outer tubes, including mixing reaction zone, crystallization reaction zone, centrifugal solidliquid separation zone, crystal return flow zone, effluent overflow area, and phosphorus collection area [20]. The crystals of porous calcium silicate hydrates can be circulated in the system, which is beneficial for effectively recovering the high concentration of phosphorus in the wastewater. The circulating crystal reactor can be used as phosphorus nuclei, which is conducive to recover low concentration of phosphorus.

In this experiment, calcium silicate hydrates were prepared with carbide residue and white carbon black. Then, it was modified by polyethylene glycol (PEG) to improve the performance of phosphorus recovery. Based on the fundamental principle of phosphorus recovery and technological characteristics of reactor, a fluidized bed of internal circulation for phosphorus crystallization was designed. The modified CSHs were characterized by X-ray diffraction (XRD), $\mathrm{N}_{2}$ adsorption-desorption isotherms, and Fourier transform infrared spectroscopy (FT-IR). The effects of initial concentration of phosphorus, P-CSHs-PEG2000 dosage, and stirring speed on recovery efficiency of phosphorus were analyzed, and the products of phosphorus recovery in this system were confirmed.

\section{Materials and Methods}

2.1. Materials. Carbide residue was used as calcium sources and obtained from Chongqing Changshou Chemical Co. Ltd. White carbon black was used as Si sources and purchased from Chongqing Jianfeng Chemical Co. Ltd. Chemical constituents of carbide residue and white carbon black were shown in Table 1 . The polyethylene glycol (PEG) regents were purchased from Chongqing Jianfeng Chemical Co. Ltd and the molecular weight of PEG was 200, 1000, 2000, 6000 , and 20000, respectively. The phosphorus solution was prepared by adding $\mathrm{KH}_{2} \mathrm{PO}_{4}$ (analytical reagent, Chongqing Boyi Chemical reagent Co. Ltd.) and the initial concentration of phosphorus was $30 \mathrm{mg} / \mathrm{L}$. All chemicals were used without further purification and all solutions were prepared using Milli-Q water.

\subsection{Preparation and Modification of Porous Calcium Silicate Hydrates}

2.2.1. Preparation of Porous Calcium Silicate Hydrates. Preparation of porous calcium silicate hydrates was shown as follows: firstly, the mixture of carbide residue and white carbon black was prepared at the molar ratios of $\mathrm{Ca} / \mathrm{Si}$ at $1.6: 1$. And the slurry was generated by dissolving the mixture with a liquid/solid ratio of 30 . Secondly, the slurry hydrothermally reacted at $170^{\circ} \mathrm{C}$ for 6 hours in reaction still and then taken out when the temperature was reduced to room temperature. Thirdly, the slurry was filtrated and dried at $105^{\circ} \mathrm{C}$ for 2 hours. Then the final products were ground and filtrated through a sieve of 200 mesh. The prepared samples were porous calcium silicate hydrates (P-CSHs) [21].

2.2.2. Modification of Porous Calcium Silicate Hydrates. The modification of porous calcium silicate hydrates was shown as follows: firstly, the prepared porous calcium silicate hydrates $(1.0 \mathrm{~g})$ were dissolved and then added to polyethylene glycol regent with different molecular weights (200, 1000, 2000, 6000 , and 20000). The solution was kept stirring at the rate of $60 \mathrm{r} / \mathrm{min}$ for 1 hour at water bath. Secondly, the slurry was filtrated and hydrothermally reacted at $500^{\circ} \mathrm{C}$ for 2 hours in muffle. After the sample cooled down to room temperature, the final products were ground and filtrated through a sieve of 200 mesh. The prepared samples were named as P-CSHs-PEG200, P-CSHs-PEG1000, P-CSHs-PEG2000, PCSHs-PEG6000, and P-CSHs-PEG20000 [22].

2.3. Experimental Section. The experiments were performed in a glass reactor with $1.0 \mathrm{~L}$ potassium dihydrogen phosphate solutions and the initial concentration of $\mathrm{KH}_{2} \mathrm{PO}_{4}$ is in the range of $5 \sim 40 \mathrm{mg} / \mathrm{L}$. The schematic diagram of internal recycle reactor was shown in Figure 1, and the operation of reactor was continuous inflow and indirect outflow. During all the experiments, the phosphorus content (\%) in the solid product was calculated by

$$
P=\frac{\left[\sum\left(c_{0}-c_{t i}\right)\right] \times v}{w} \times 100 \%,
$$

where $C_{t i}$ is the phosphorus concentration at a given time, $\mathrm{mg} / \mathrm{L} ; C_{0}$ is the phosphorus concentration at the initial time, $\mathrm{mg} / \mathrm{L} ; \mathrm{w}$ is the weight of solid product, mg; $v$ is the volume of solution, $\mathrm{L}$.

2.4. Analytical Method. The crystal structures and phase compositions of the samples were characterized by X-ray 

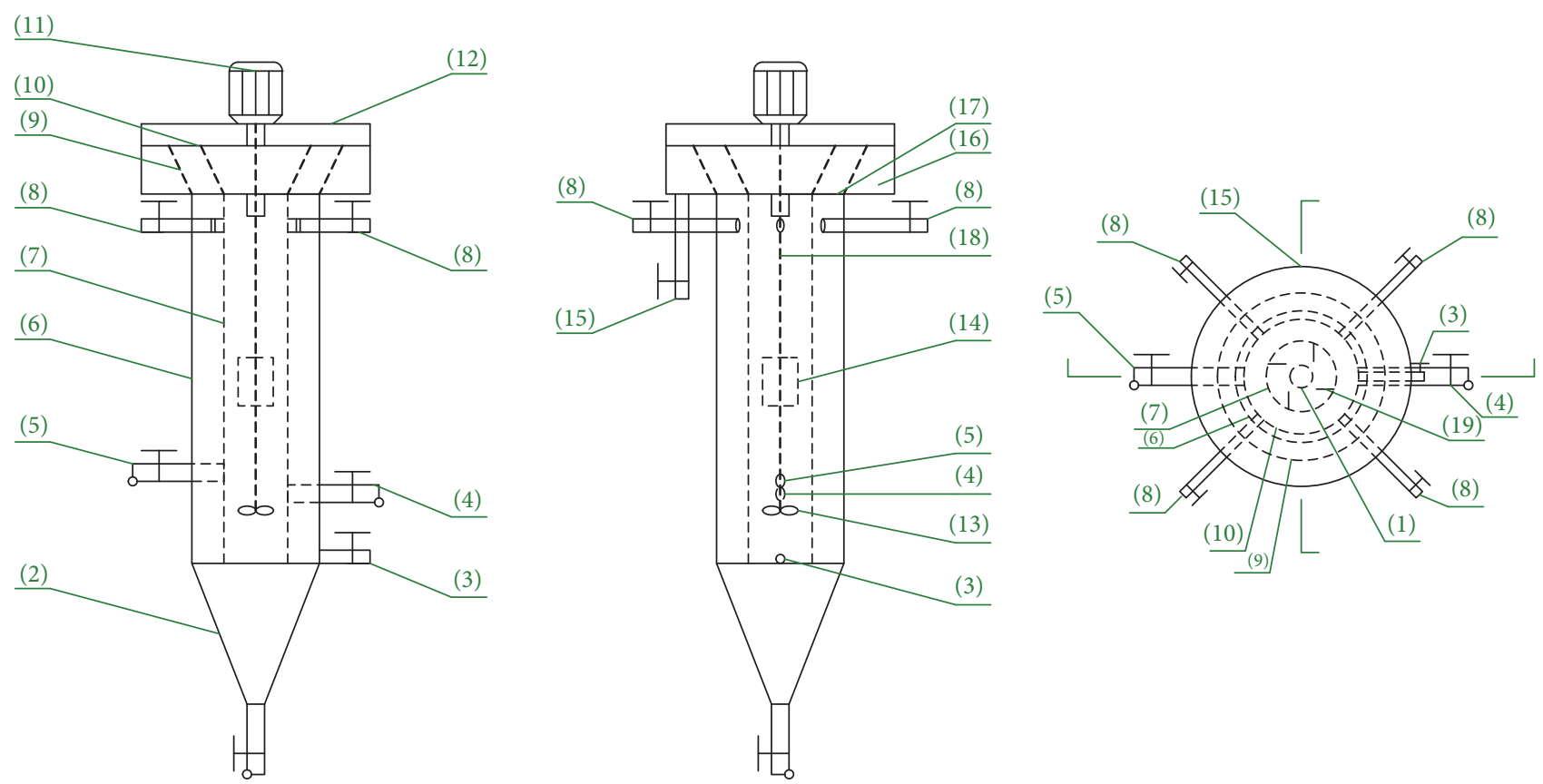

FIGURE 1: The schematic diagram of internal recycle reactor for phosphorus recovery. (1) Outlet of product; (2) collecting bowl for crystalline particle; (3) sampling port; (4) water inlet; (5) feed inlet; (6) outer tube; (7) inner tube; (8) reagent addition; (9) outer overflow nozzle; (10) internal overflow nozzle; (11) electrical machine; (12) mounting plate of electrical machine; (13) spiral stirrer; (14) rectangle stirrer; (15) water outlet; (16) effluent flume; (17) reflowing valve; (18) transmission shaft; (19) baffle of turbulent flow.

diffraction (XRD) on a D/Max IIIB X-ray powder diffractometer (Rigaku, Japan) with $\mathrm{Cu} \mathrm{K} \alpha$ radiation. The specific surface area and pore-size distribution of the as-prepared samples were calculated by $\mathrm{N}_{2}$ adsorption-desorption analysis (ASAP2020, Micromeritics, USA). The chemical bonds of samples were analyzed by the Fourier transform infrared spectroscopy (FT-IR) (Nexus 670). Morphology, microstructure, and size of products were measured by scanning electronic microscopy (SEM, JEM-6490) [23]. The concentration of dissolved $\mathrm{Ca}^{2+}$ ion was measured using a 710 series inductively coupled plasma optical emission spectrometer (ICP-OES, Agilent Technology, USA).

\section{Results and Discussion}

3.1. XRD Characterization of the Modified CSHs. The XRD spectra of porous calcium silicate hydrates modified by PEG with different molecular weights were shown in Figure 2. Before CSHs modification, the diffraction peaks with $2 \theta=$ $8.40^{\circ}, 29.92^{\circ}, 31.78^{\circ}, 35.74^{\circ}$, and $49.70^{\circ}$ (JCPDS card No. $25-$ 1464) are observed [24]. The characteristic peaks of the modified CSHs samples at $2 \theta=31.78^{\circ}$ and $35.74^{\circ}$ have disappeared obviously, and the strongest characteristic peaks at $2 \theta=29.92^{\circ}$ have weakened. The addition of PEG results in distortion of the crystalline structure and reduction of the crystallinity of porous calcium silicate hydrates [25]. The diffraction peaks of porous calcium silicate hydrates with $2 \theta=8.40^{\circ}$ are according to (002), which almost disappear after modified by PEG. The PEG reagent enters the interlayer of porous calcium

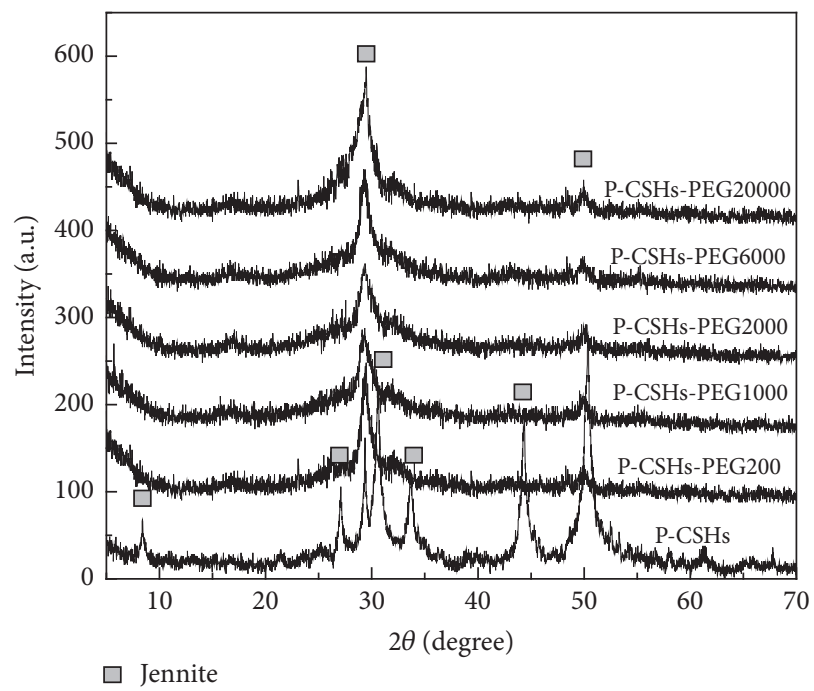

FIGURE 2: The XRD patterns of porous calcium silicate hydrates modified by PEG with different molecular weights.

silicate hydrates and increases the interlayer space of porous calcium silicate hydrates. The disappearing of diffraction peaks shows that the degree of crystallinity of porous calcium silicate hydrates is decreased significantly. The fitting of MDI jade5.0 indicates that the crystallinity of P-CSHs, 
TABLE 2: The data of pore structure for different materials.

\begin{tabular}{lccc}
\hline Samples & Pore volume $/\left(\mathrm{cm}^{3} / \mathrm{g}\right)$ & Pore diameter $/(\mathrm{nm})$ & $S_{\mathrm{BET}} /\left(\mathrm{m}^{2} / \mathrm{g}\right)$ \\
\hline P-CSHs-PEG200 & 0.24 & 14.90 & 64.00 \\
P-CSHs-PEG1000 & 0.24 & 14.85 & 65.74 \\
P-CSHs-PEG2000 & 0.33 & 15.43 & 87.48 \\
P-CSHs-PEG6000 & 0.20 & 10.28 & 77.95 \\
P-CSHs-PEG20000 & 0.23 & 20.35 & 46.03 \\
P-CSHs & 0.29 & 26.37 & 43.97 \\
\hline
\end{tabular}

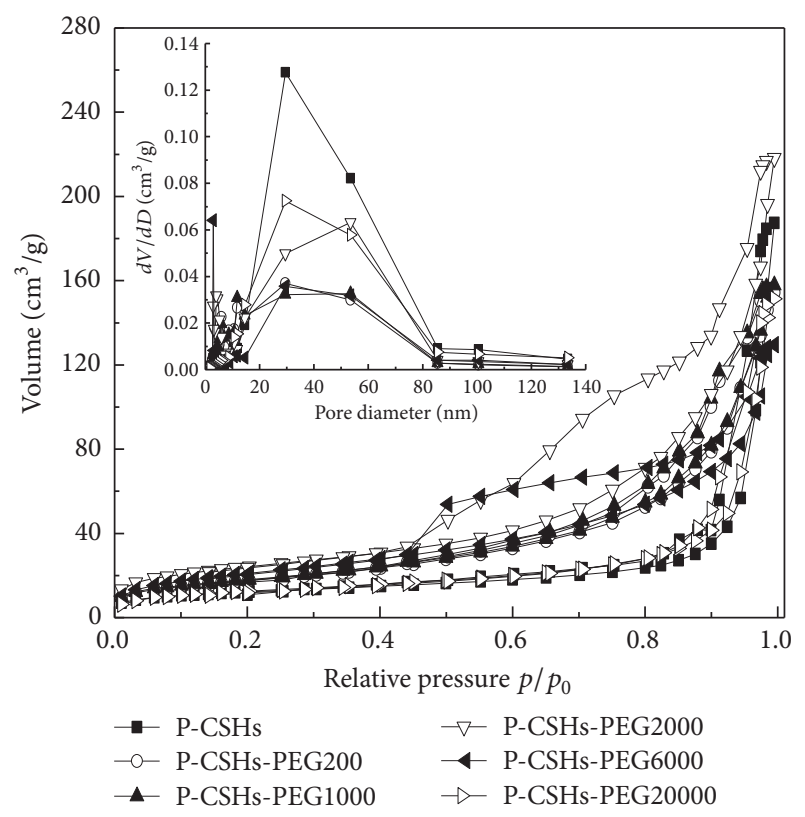

FIgURE 3: The $\mathrm{N}_{2}$ adsorption-desorption isotherms and pore-size distribution curves for different materials.

P-CSHs-PEG200, P-CSHs-PEG1000, P-CSHs-PEG2000, PCSHs-PEG6000, and P-CSHs-PEG20000 is $43.37 \%, 26.89 \%$, $24.9 \%, 16.94 \%, 18.24 \%$, and $38.16 \%$, respectively.

3.2. Nitrogen Adsorption-Desorption Analysis. The $\mathrm{N}_{2}$ adsorption-desorption isotherms and pore-size distribution curves for different materials were shown in Figure 3. The phenomena of hysteresis loop in $\mathrm{N}_{2}$ adsorption-desorption isotherms for different materials are caused by the condensation of pores [26]. The data of pore structure of various materials is shown in Table 2. The specific surface area and pore volume of porous calcium silicate hydrates are increased by the modification of PEG. However, when the molecular weight of PEG is too low or too high, the surface area and pore volume of porous calcium silicate hydrates are not obviously changed. Therefore, when the porous calcium silicate hydrates are modified by PEG at molecular weight of 2000 , it has the largest specific surface area of $87.48 \mathrm{~m}^{2} / \mathrm{g}$ and pore volume of $0.33 \mathrm{~cm}^{3} / \mathrm{g}$.

3.3. FT-IR Analysis. In order to investigate the chemical bonding of the samples, the calcium silicate hydrates before

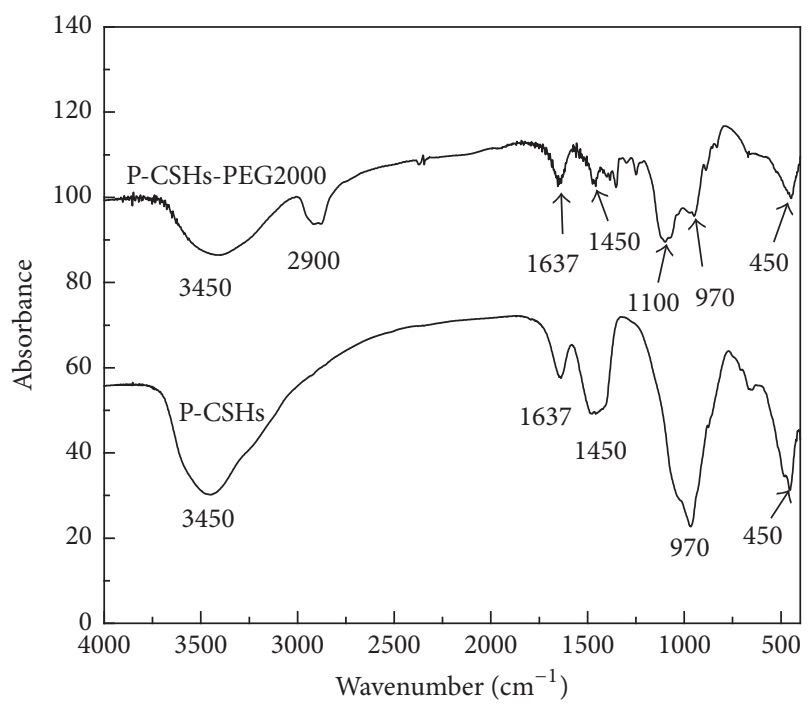

FIGURE 4: FT-IR spectra of calcium silicate hydrates before and after modification.

and after modification were analyzed by FT-IR. As shown in Figure 4, there are a series of absorption peaks at $3450 \mathrm{~cm}^{-1}$ $\left(\mathrm{OH}^{-}\right), 970 \mathrm{~cm}^{-1}$ (Si-O-Si), $1637 \mathrm{~cm}^{-1}(\mathrm{O}-\mathrm{H}), 1450 \mathrm{~cm}^{-1}$ (C$\mathrm{O}-\mathrm{H})$, and $450 \mathrm{~cm}^{-1}$ (Si-O-Si) in FT-IR spectra of two samples [27]. The characteristic peaks are ascribed to the silicon oxygen tetrahedron structure of calcium silicate hydrates. However, the intensity of characteristic peaks for P-CSHsPEG2000 sample is obviously weaker than calcium silicate hydrates, indicating that the order degree of calcium silicate hydrates is decreased with the addition of PEG. In addition, P-CSHs-PEG2000 samples exhibit a stretching vibration peak of $\mathrm{C}-\mathrm{H}$ at $2900 \mathrm{~cm}^{-1}$ and a stretching vibration absorption peak of $\mathrm{C}-\mathrm{O}$ at $1100 \mathrm{~cm}^{-1}$. These characteristic peaks indicate that $\mathrm{PEG}$ is a polymer with $-\mathrm{CH}_{2}-\mathrm{CH}_{2}-\mathrm{O}$ - group in the main chain of molecules and belongs to nonionic dispersant [28]. The molecular structure consists of two parts: anchoring group and solvation chain. Before modification, the surface structure of calcium silicate hydrates is relatively dense and the molecular structure is closely arranged. When PEG is added to the slurry of calcium silicate hydrates, the polymer enters the defect site of the silicon oxygen tetrahedron chain of the calcium silicate hydrates or enters the interlayer. The anchoring groups are adsorbed on the surface of calcium silicate hydrates. And the solvation chains are expanded in 


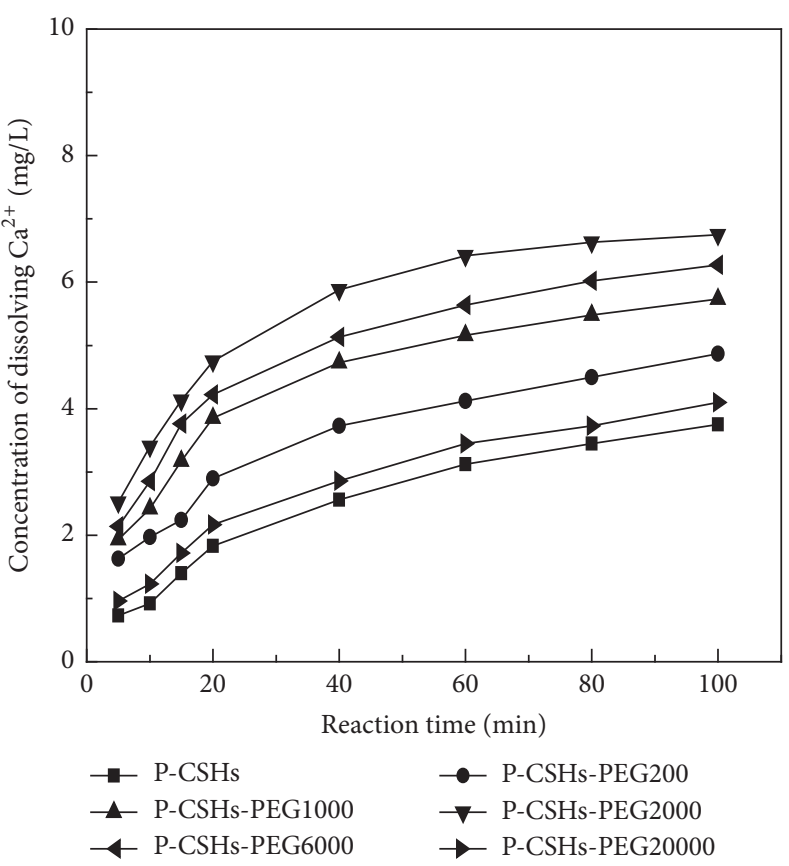

(a)

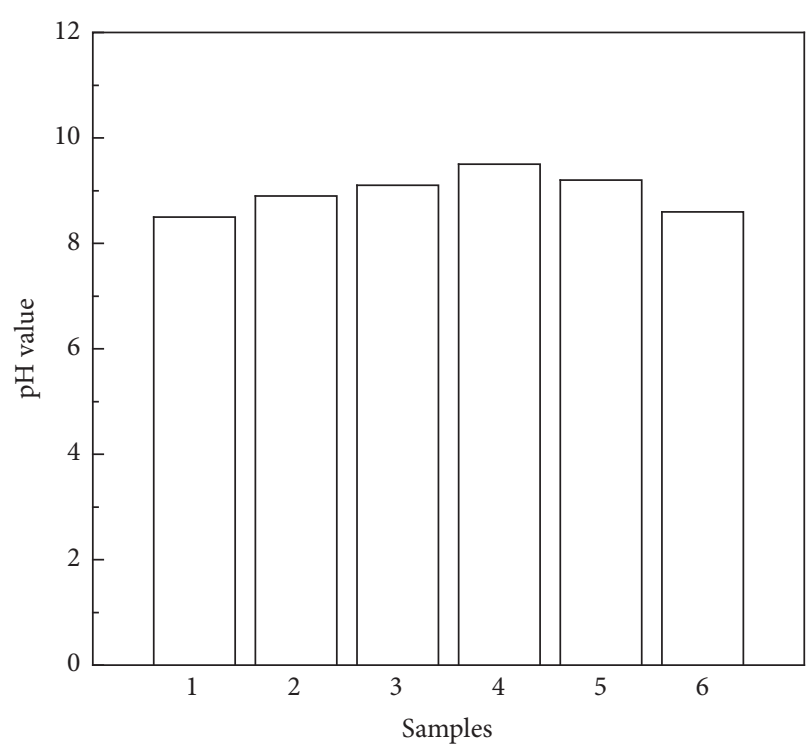
(1) $\mathrm{P}-\mathrm{CSHs}$
(3) P-CSHs-PEG1000
(5) P-CSHs-PEG6000
(2) P-CSHs-PEG200
(4) P-CSHs-PEG2000
(6) P-CSHs-PEG20000

(b)

Figure 5: (a) The variation of dissolved $\mathrm{Ca}^{2+}$ concentration for different samples; (b) the variation of $\mathrm{pH}$ value in solution.

the medium to form a barrier layer, which prevents the aggregation of solid particles and achieves the function of steric hindrance. PEG is burned away by calcinations and generated more pore structure.

\subsection{The Analysis of Dissolving Property for Calcium Silicate} Hydrates. The concentration of dissolved $\mathrm{Ca}^{2+}$ and the variation of $\mathrm{pH}$ for different samples were shown in Figure 5. The modified calcium silicate hydrates can increase the concentration of dissolved $\mathrm{Ca}^{2+}$ and $\mathrm{pH}$ of solution [29]. Comparing with the P-CSHs sample, the concentration of dissolved $\mathrm{Ca}^{2+}$ from the modified P-CSHs-PEG2000 has been increased from $3.75 \mathrm{mg} / \mathrm{L}$ to $6.75 \mathrm{mg} / \mathrm{L}$ when it reached the reaction equilibrium. However, when the molecular weight of PEG is too large or too small, the calcium solubility of the modified calcium silicate hydrates is not significantly increased. The concentration of dissolved $\mathrm{Ca}^{2+}$ for the P-CSHs-PEG20000 is only $4.10 \mathrm{mg} / \mathrm{L}$ (Figure 5(a)). The modified calcium silicate hydrates can increase the $\mathrm{pH}$ of solution [30]. When various materials are reached to the solubility equilibrium, the $\mathrm{pH}$ value of solution is basically maintained at 8.6 9.5. Among them, the P-CSHs-PEG2000 samples can increase the $\mathrm{pH}$ value of solution to 9.5 (Figure 5(b)). The high concentration of dissolved $\mathrm{Ca}^{2+}$ and high $\mathrm{pH}$ value of solution are beneficial for phosphorus recovery. Therefore, the P-CSHs-PEG2000 samples are used as the material for phosphorus recovery in internal recycle reactor. Therefore, the P-CSHs-PEG2000 samples are used as the material for phosphorus recovery in internal recycle reactor.

\subsection{The Analysis of Operation Efficiency for Phosphorus Recovery}

3.5.1. The Effect of P-CSHs-PEG2000 Dosage on Phosphorus Recovery System. The effect of P-CSHs-PEG2000 dosage on phosphorus recovery system was investigated. While keeping parameters such as initial concentration of phosphorus and stirring speed fixed, the phosphorus recovery system was performed using different dosages of P-CSHs-PEG2000 $(0.5,1,2,3$, and $4 \mathrm{~g} / \mathrm{L})$. As shown in Figure 6, when the dosages of P-CSHs-PEG2000 are changed in the range of $1 \sim 4 \mathrm{~g} / \mathrm{L}$, the residual concentration of phosphorus remained at $4 \sim 5 \mathrm{mg} / \mathrm{L}$ after $60 \mathrm{~min}$ reaction. When the dosage of P-CSHs-PEG2000 is too small, the residual concentration of phosphorus is increased significantly and remained at $9.44 \mathrm{mg} / \mathrm{L}$. The results are different from the conventional phenomena of coagulation and sedimentation. Comparing the traditional coagulation and sedimentation process, when the initial concentration of phosphorus is the same, the more dosage of the reagent can increase the recovery efficiency of phosphorus. However, the principle of phosphorus recovery in this system is different from the traditional principles of coagulation and sedimentation. In conventional coagulation and sedimentation process, the recovery efficiency of phosphorus mainly depends on the alkalinity generated by adding excess chemicals, which results in the lower utilization of agent for phosphorus recovery. Considering the effective utilization of P-CSHs-PEG2000 samples, the optimum dosage of crystal for phosphorus recovery is $1.0 \mathrm{~g} / \mathrm{L}$. 


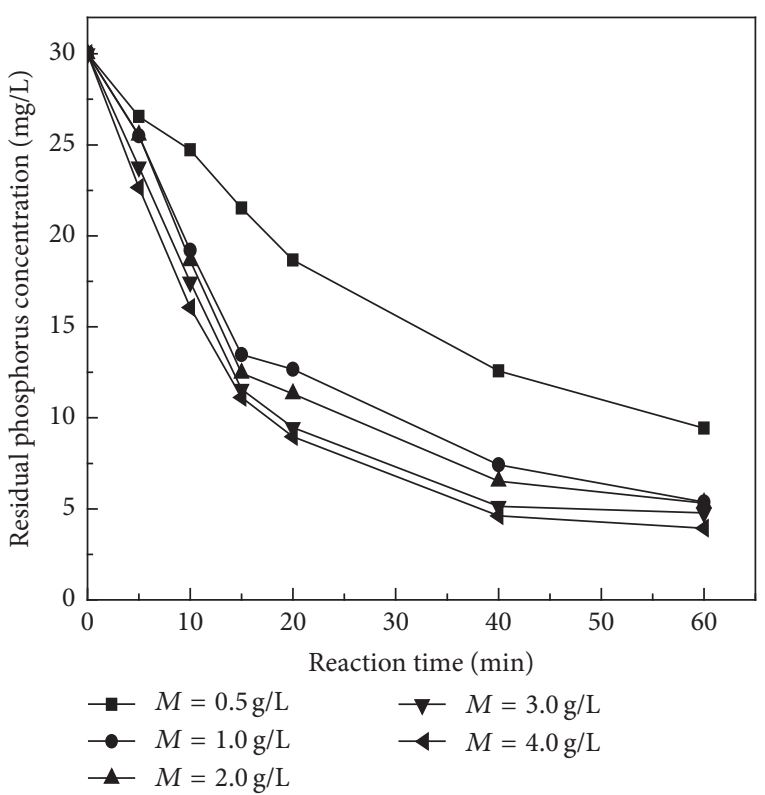

Figure 6: The effect of P-CSHs-PEG2000 dosage on phosphorus recovery system (initial concentration of phosphorus, $30 \mathrm{mg} / \mathrm{L}$; initial $\mathrm{pH}, 7.0$; stirring speed, $60 \mathrm{r} / \mathrm{min}$ ).

\subsubsection{The Effect of Stirring Speed on Phosphorus Recovery} System. The effect of stirring speed on phosphorus recovery system was investigated. While keeping parameters such as initial concentration of phosphorus and P-CSHs-PEG2000 dosage fixed, the phosphorus recovery system was performed using different stirring speed $(40,60,80,100$, and $120 \mathrm{r} / \mathrm{min})$. As shown in Figure 7, the stirring speed has a greater influence on residual concentration of phosphorus. When the stirring speed is $80 \mathrm{r} / \mathrm{min}$, the residual concentration of phosphorus reaches the minimum $(3.93 \mathrm{mg} / \mathrm{L})$. However, when the stirring speeds are too higher or too lower, this will affect the operation efficiency and increase the residual concentration of phosphorus. In the process of phosphorus recovery, hydroxyapatites are generated on the surface of porous calcium silicate hydrates. The lower stirring speed cannot make the crystal fully contact with the sewage and the higher stirring speed cannot make the hydroxyapatites grow well on the surface of the crystal. Therefore, the best stirring speed for phosphorus recovery is $80 \mathrm{r} / \mathrm{min}$.

3.5.3. The Effect of Initial Concentration of Phosphorus on Operation Efficiency. The effect of initial concentration of phosphorus on operation efficiency was investigated. While keeping parameters such as P-CSHs-PEG2000 dosage and stirring speed fixed, the phosphorus recovery system was performed using different concentrations of phosphorus (40, $30,20,10$, and $5 \mathrm{mg} / \mathrm{L}$ ). As shown in Figure 8 , the initial concentration of phosphorus can only determine the rate of reaction; however, it cannot change the chemical equilibrium. When the reaction equilibrium is reached, the residual phosphorus concentration is basically stable between 3.5 $4.0 \mathrm{mg} / \mathrm{L}$. With respect to phosphorus treatment, the effluent of phosphorus concentration is not up to the standard in

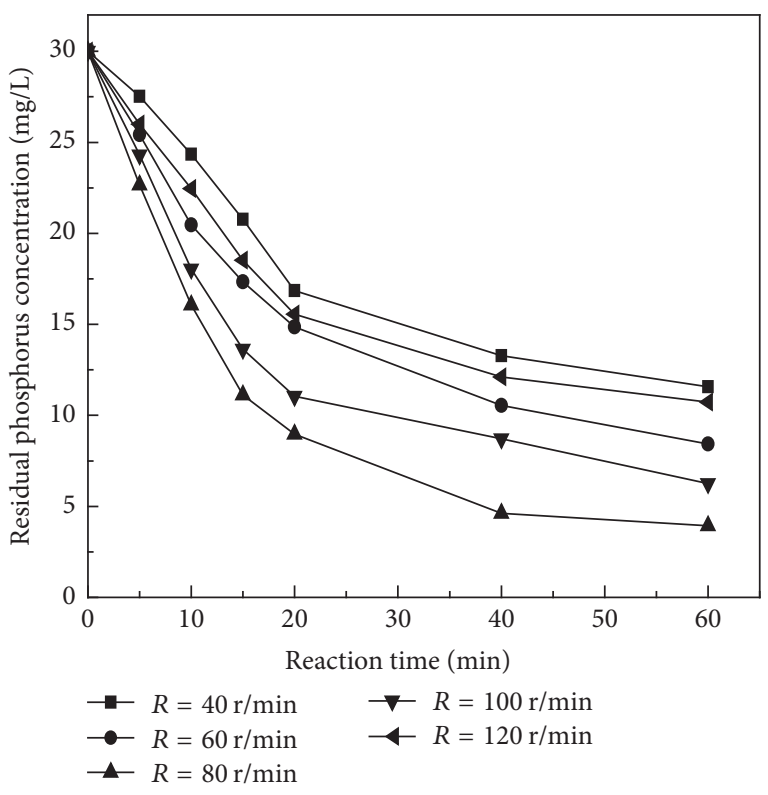

FIgURE 7: The effect of stirring speed on phosphorus recovery system (initial concentration of phosphorus, $30 \mathrm{mg} / \mathrm{L}$; initial $\mathrm{pH}$, 7.0; P-CSHs-PEG2000 dosage, $1.0 \mathrm{~g} / \mathrm{L}$ ).

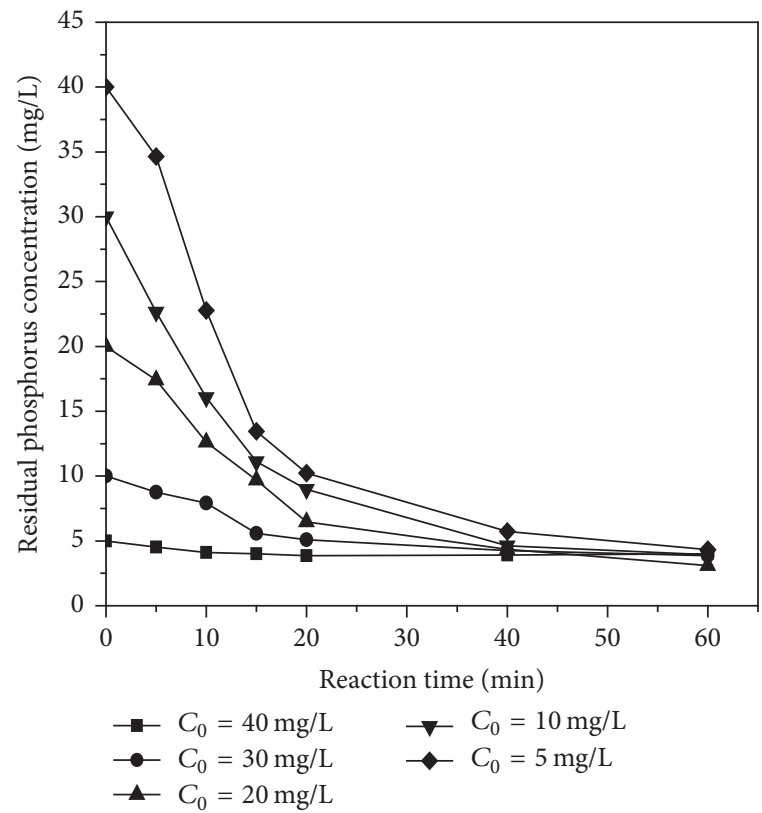

FIgURE 8: The effect of initial concentration of phosphorus on operation efficiency (initial pH, 7.0; P-CSHs-PEG2000 dosage, $1.0 \mathrm{~g} / \mathrm{L}$; stirring speed, $80 \mathrm{r} / \mathrm{min}$ ).

this system. But from the point of phosphorus recovery, the increase of phosphorus concentration is beneficial to improve the phosphorus recovery efficiency. Therefore, the initial concentration of phosphorus for phosphorus recovery is $40 \mathrm{mg} / \mathrm{L}$.

3.6. The Characterization of Deposition. The residual concentration of phosphorus changed greatly in the four use 


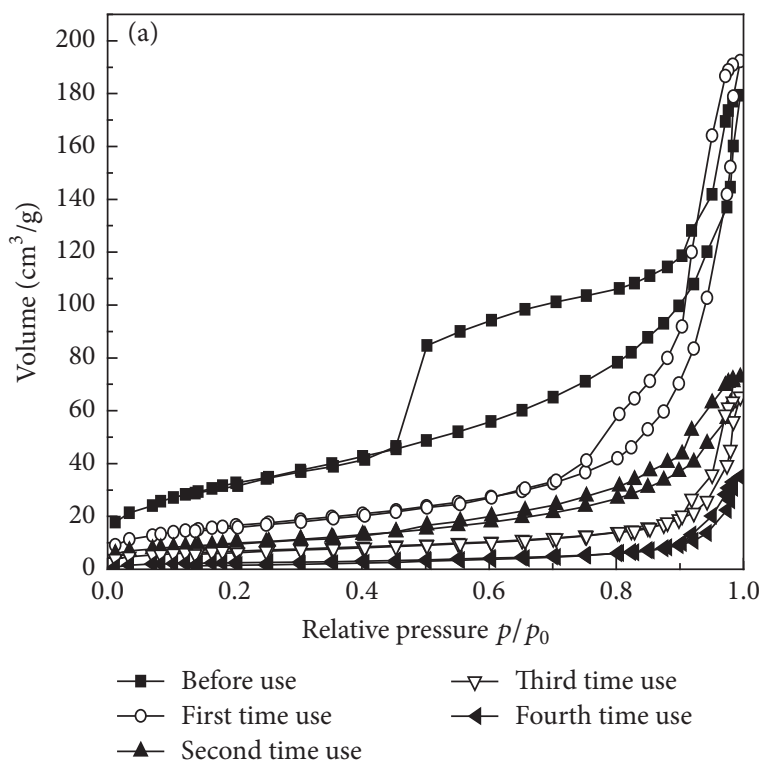

(a)

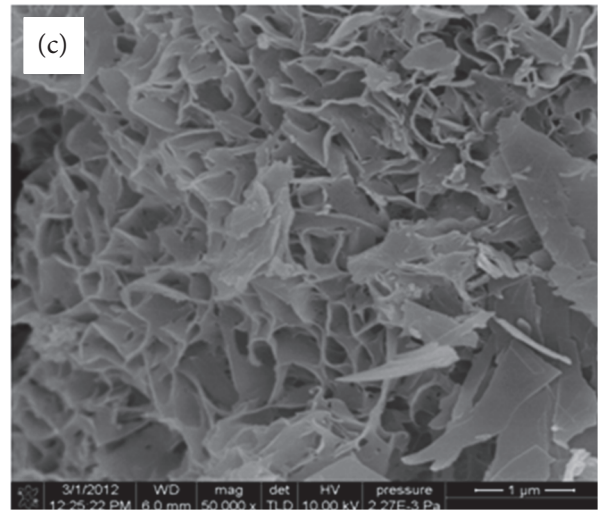

(c)

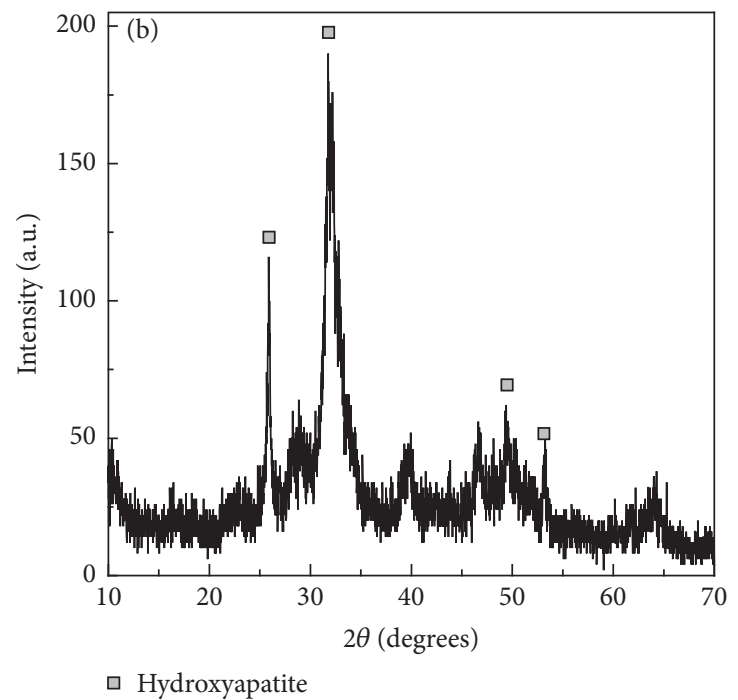

(b)

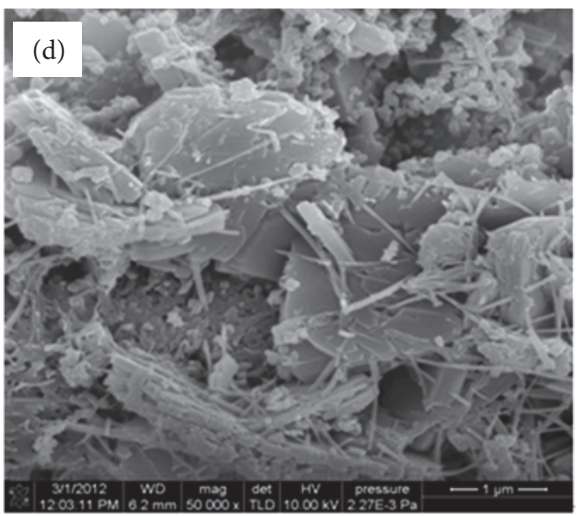

(d)

FIGURE 9: The characterization of deposition: (a) the $\mathrm{N}_{2}$ adsorption-desorption isotherms for different times of using P-CSHs-PEG2000; (b) XRD patterns of deposit; (c) SEM images of P-CSHs-PEG2000 before use; (d) SEM images of P-CSHs-PEG2000 after four times use.

times of P-CSHs-PEG2000 samples. Therefore, the influence of use times on pore structure of P-CSHs-PEG2000 samples was investigated. As shown in Figure 9(a), the mesoporous adsorption mainly occurred in the middle pressure zone $\left(0.4<p / p_{0}<0.9\right)$. In this region, the phenomenon of hysteresis loop in various samples gradually disappeared and the adsorption curves are gradually declined with the increasing times of using calcium silicate hydrates. The data of pore volume and surface area about P-CSHs-PEG2000 samples after different use times are shown in Table 3.

With increasing the use times of P-CSHs-PEG2000 samples for phosphorus recovery, the pore volume and surface area are significantly reduced. The structural properties of $\mathrm{P}$ CSHs-PEG2000 samples used for phosphorus recovery were further investigated by XRD in the range of $10-70^{\circ}$. As shown in Figure 9(b), the pronounced diffraction peaks in the XRD pattern at $2 \theta$ of $26.4^{\circ}, 33.4^{\circ}, 50.7^{\circ}$, and $77.52^{\circ}$ corresponded to hydroxylapatite in accordance with the standard [31] and
TABLE 3: The data of pore structure for P-CSHs-PEG2000 samples after different use times.

\begin{tabular}{lcc}
\hline Samples & Pore volume $/\left(\mathrm{cm}^{3} / \mathrm{g}\right)$ & $S_{\mathrm{BET}} /\left(\mathrm{m}^{2} / \mathrm{g}\right)$ \\
\hline Before use & 0.33 & 87.48 \\
First time use & 0.25 & 55.00 \\
Second time use & 0.14 & 35.01 \\
Third time use & 0.10 & 14.90 \\
Fourth time use & 0.05 & 3.75 \\
\hline
\end{tabular}

the characteristic peaks of porous calcium silicate hydrate disappeared basically. Indicating porous calcium silicate hydrate is mainly covered by hydroxyapatite and achieves phosphorus recovery. The surface variation of porous calcium silicate hydrate in the process of phosphorus recovery is analyzed by SEM. As shown in Figures 9(c)-9(d), before PCSHs-PEG2000 samples are used for phosphorus recovery, 


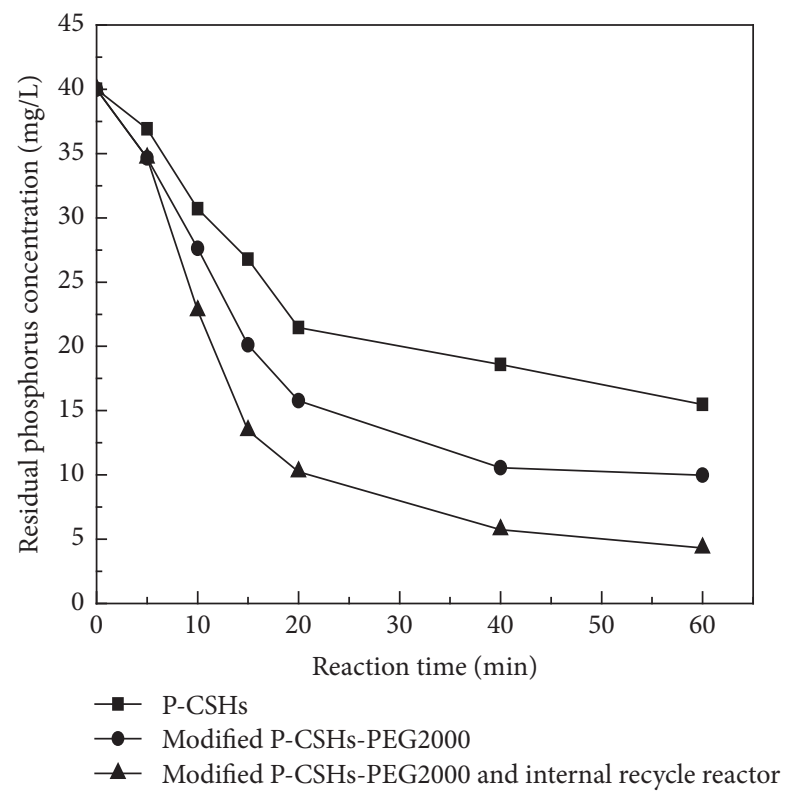

FIGURE 10: Comparing the three systems for phosphorus recovery (initial concentration of phosphorus, $40 \mathrm{mg} / \mathrm{L}$; initial pH, 7.0; dosage of crystal, $1.0 \mathrm{~g} / \mathrm{L}$; stirring speed, $80 \mathrm{r} / \mathrm{min}$ ).

it obviously has a porous structure [32]. After four times using P-CSHs-PEG2000 samples for phosphorus recovery, it has no obvious pore structure and a large amount of flocculent substance appears. According to the analysis of $\mathrm{XRD}$, the deposition on the surface of calcium silicate hydrate is hydroxyapatite. The phosphorus content of the deposition was $14.43 \%$, which is basically closed to the phosphorus content of phosphorus minerals (15\%). Therefore, it provides a simple and beneficial method for phosphorus recovery from the electroplating wastewater.

3.7. The Process of Phosphorus Recovery in Internal Recycle Reactor. Under the optimum conditions, the large surface area of porous calcium silicate hydrate quickly makes the materials dissolve calcium and effectively maintain the high concentrations of $\mathrm{Ca}^{2+}$ and $\mathrm{OH}^{-}$, which makes the $\mathrm{pH}$ of the solution kept at 8.5 9.5. Under this $\mathrm{pH}$ condition, the existence form of phosphorus is $\mathrm{HPO}_{4}{ }^{2-}$. Thus, high concentrations of $\mathrm{Ca}^{2+}, \mathrm{OH}^{-}$, and $\mathrm{HPO}_{4}{ }^{2-}$ formed, which facilitates the formation of hydroxyapatite in a weak alkaline environment [33]. As shown in Figure 10, comparing the three systems (i.e., P-CSHs, modified P-CSHs-PEG2000, and the modified P-CSHs-PEG2000 combined with the internal recycle reactor) for phosphorus recovery, the internal recycle reactor enhances porous calcium silicate hydrates to recover phosphorus from electroplating wastewater.

The internal recycle reactor for phosphorus recovery is structured with an inner cylinder and an external cylinder, wherein the inner cylinder consisted of mixing reaction zone, crystallization reaction zone, and solid-liquid separation zone [34]. The external cylinder includes hydroxyapatite formation zone and crystallization settling zone. In addition, the top of the reactor is the effluent settling zone and the bottom of the reactor is the crystal collecting zone. The design of double cylinder structure makes the reactor efficiently recover phosphorus. The operation way of reactor is simple and the operating cost is low. The functions of different reaction zones are introduced as follows: the mixed reaction zone makes the porous calcium silicate hydrate and phosphorus wastewater in full contact; the crystallization reaction zone makes the phosphate rapidly form hydroxyapatite; the solidliquid separation zone makes the treated wastewater overflow from the top of the reactor and the porous calcium silicate hydrates get into the external cylinder under centrifugal force; the growth of hydroxyapatite is based on the principle of secondary nucleation, which makes the hydroxyapatite continually grow. The cyclic precipitation zone allows the hydroxyapatite to flow back to the inner cylinder and improves phosphorus recovery; the collection zone is used for deposition and collection products of phosphorus recovery [35]. Therefore, the design of double cylinder structure makes the reactor efficiently recover phosphorus.

\section{Conclusion}

The porous calcium silicate hydrates could be circulated in the internal recycle reactor, which could effectively recover high concentration of phosphorus from electroplating wastewater. Compared with different samples, the modified P-CSHs-PEG2000 sample had larger specific surface area and higher pore volume, which was beneficial for phosphorus recovery. Moreover, the dissolution rate of $\mathrm{Ca}^{2+}$ from PCSH-PEG2000 was fast, which was favorable for phosphorus precipitation and phosphorus recovery. The effects of initial concentration of phosphorus, P-CSHs-PEG2000 dosage, and stirring speed on phosphorus recovery were analyzed, so the optimal operation conditions for phosphorus recovery were obtained. The deposition was analyzed by XRD, $\mathrm{N}_{2}$ adsorption-desorption, and SEM techniques; it was indicated that the pore volume and surface area of the P-CSHsPEG2000 were significantly reduced, and the deposition on the surface of P-CSHs-PEG2000 was hydroxyapatite. The main products in the process of phosphorus recovery were hydroxyapatite and the phosphorus content of the products was $14.43 \%$, which is basically closed to the phosphorus content of phosphorus minerals (15\%).

\section{Conflicts of Interest}

The authors declare that they have no conflicts of interest.

\section{References}

[1] F. V. Hackbarth, D. Maass, A. A. U. de Souza, V. J. P. Vilar, and S. M. A. G. U. de Souza, "Removal of hexavalent chromium from electroplating wastewaters using marine macroalga Pelvetia canaliculata as natural electron donor," Chemical Engineering Journal, vol. 290, pp. 477-489, 2016.

[2] Z. Rajabalizadeh and D. Seifzadeh, "Strontium phosphate conversion coating as an economical and environmentally-friendly pretreatment for electroless plating on AM60B magnesium alloy," Surface and Coatings Technology, vol. 304, pp. 450-458, 2016. 
[3] N. P. Springer, "Physical, technical, and economic accessibility of resources and reserves need to be distinguished by grade: application to the case of phosphorus," Science of the Total Environment, vol. 577, pp. 319-328, 2017.

[4] Y. Wang, Y. Yu, H. Li, and C. Shen, "Comparison study of phosphorus adsorption on different waste solids: Fly ash, red mud and ferric-alum water treatment residues," Journal of Environmental Sciences (China), vol. 50, pp. 79-86, 2016.

[5] K. Zhou, M. Barjenbruch, C. Kabbe, G. Inial, and C. Remy, "Phosphorus recovery from municipal and fertilizer wastewater: China's potential and perspective," Journal of Environmental Sciences (China), vol. 52, pp. 151-159, 2017.

[6] M. Hupfer, K. Reitzel, A. Kleeberg, and J. Lewandowski, "Longterm efficiency of lake restoration by chemical phosphorus precipitation: scenario analysis with a phosphorus balance model," Water Research, vol. 97, pp. 153-161, 2016.

[7] C. Wan, S. Ding, C. Zhang et al., "Simultaneous recovery of nitrogen and phosphorus from sludge fermentation liquid by zeolite adsorption: mechanism and application," Separation and Purification Technology, vol. 180, pp. 1-12, 2017.

[8] Y. Zhang, W. Zhang, and B. Pan, "Struvite-based phosphorus recovery from the concentrated bioeffluent by using HFO nanocomposite adsorption: effect of solution chemistry," Chemosphere, vol. 141, pp. 227-234, 2015.

[9] K. Asoh and M. Ebihara, "Accurate determination of trace amounts of phosphorus in geological samples by inductively coupled plasma atomic emission spectrometry with ion-exchange separation," Analytica Chimica Acta, vol. 779, pp. 8-13, 2013.

[10] H. Huang, D. Zhang, Z. Zhao, P. Zhang, and F. Gao, "Comparison investigation on phosphate recovery from sludge anaerobic supernatant using the electrocoagulation process and chemical precipitation," Journal of Cleaner Production, vol. 141, pp. 429438, 2017.

[11] E. R. Hall, A. Monti, and W. W. Mohn, "A comparison of bacterial populations in enhanced biological phosphorus removal processes using membrane filtration or gravity sedimentation for solids-liquid separation," Water Research, vol. 44, no. 9, pp. 2703-2714, 2010.

[12] L.-C. Hua, C. Huang, Y.-C. Su, T.-N. Nguyen, and P.-C. Chen, "Effects of electro-coagulation on fouling mitigation and sludge characteristics in a coagulation-assisted membrane bioreactor," Journal of Membrane Science, vol. 495, pp. 29-36, 2015.

[13] M. L. Gerardo, N. H. M. Aljohani, D. L. Oatley-Radcliffe, and R. W. Lovitt, "Moving towards sustainable resources: Recovery and fractionation of nutrients from dairy manure digestate using membranes," Water Research, vol. 80, pp. 80-89, 2015.

[14] Y.-J. Tu and C.-F. You, "Phosphorus adsorption onto green synthesized nano-bimetal ferrites: Equilibrium, kinetic and thermodynamic investigation," Chemical Engineering Journal, vol. 251, pp. 285-292, 2014.

[15] X. C. Chen, H. N. Kong, D. Y. Wu, X. Z. Wang, and Y. Y. Lin, "Phosphate removal and recovery through crystallization of hydroxyapatite using xonotlite as seed crystal," Journal of Environmental Sciences, vol. 21, no. 5, pp. 575-580, 2009.

[16] H. Yin, M. Kong, and C. Fan, "Batch investigations on P immobilization from wastewaters and sediment using natural calcium rich sepiolite as a reactive material," Water Research, vol. 47, no. 13, pp. 4247-4258, 2013.

[17] D. Xu, S. Ding, Q. Sun, J. Zhong, W. Wu, and F. Jia, "Evaluation of in situ capping with clean soils to control phosphate release from sediments," Science of the Total Environment, vol. 438, pp. 334-341, 2012.

[18] C. Li, H. Yu, S. Tabassum et al., "Effect of calcium silicate hydrates $(\mathrm{CSH})$ on phosphorus immobilization and speciation in shallow lake sediment," Chemical Engineering Journal, vol. 317, pp. 844-853, 2017.

[19] W. Guan, F. Ji, D. Fang et al., "Porosity formation and enhanced solubility of calcium silicate hydrate in hydrothermal synthesis," Ceramics International, vol. 40, no. 1, pp. 1667-1674, 2014.

[20] Z. Liu, Q. Zhao, D.-J. Lee, and N. Yang, "Enhancing phosphorus recovery by a new internal recycle seeding MAP reactor," Bioresource Technology, vol. 99, no. 14, pp. 6488-6493, 2008.

[21] S. Wang, X. Peng, Z. Tao, L. Tang, and L. Zeng, "Influence of drying conditions on the contact-hardening behaviours of calcium silicate hydrate powder," Construction and Building Materials, vol. 136, pp. 465-473, 2017.

[22] O. Mendoza, C. Giraldo, S. S. Camargo, and J. I. Tobón, "Structural and nano-mechanical properties of Calcium Silicate Hydrate (C-S-H) formed from alite hydration in the presence of sodium and potassium hydroxide," Cement and Concrete Research, vol. 74, pp. 88-94, 2015.

[23] W. Guan and X. Zhao, "Fluoride recovery using porous calcium silicate hydrates via spontaneous $\mathrm{Ca}^{2+}$ and $\mathrm{OH}^{-}$release," Separation and Purification Technology, vol. 165, pp. 71-77, 2016.

[24] K. Okano, S. Miyamaru, A. Kitao et al., "Amorphous calcium silicate hydrates and their possible mechanism for recovering phosphate from wastewater," Separation and Purification Technology, vol. 144, pp. 63-69, 2015.

[25] F. Pelisser, P. J. P. Gleize, and A. Mikowski, "Structure and micronanomechanical characterization of synthetic calcium-silicatehydrate with Poly(Vinyl Alcohol)," Cement and Concrete Composites, vol. 48, pp. 1-8, 2014.

[26] J. Zhao, Y.-J. Zhu, J. Wu et al., "Chitosan-coated mesoporous microspheres of calcium silicate hydrate: environmentally friendly synthesis and application as a highly efficient adsorbent for heavy metal ions," Journal of Colloid and Interface Science, vol. 418, pp. 208-215, 2014.

[27] S. C. Mojumdar and L. Raki, "Preparation, thermal, spectral and microscopic studies of calcium silicate hydrate-poly(acrylic acid) nanocomposite materials," Journal of Thermal Analysis and Calorimetry, vol. 85, no. 1, pp. 99-105, 2006.

[28] X. Zhang, H. Liu, Z. Huang et al., "Preparation and characterization of the properties of polyethylene glycol @ Si3N4 nanowires as phase-change materials," Chemical Engineering Journal, vol. 301, pp. 229-237, 2016.

[29] T. Missana, M. García-Gutiérrez, M. Mingarro, and U. Alonso, "Analysis of barium retention mechanisms on calcium silicate hydrate phases," Cement and Concrete Research, vol. 93, pp. 816, 2017.

[30] A. A. Rouff, N. Ma, and A. B. Kustka, "Adsorption of arsenic with struvite and hydroxylapatite in phosphate-bearing solutions," Chemosphere, vol. 146, pp. 574-581, 2016.

[31] Y. Kim and Y. J. Lee, "Characterization of mercury sorption on hydroxylapatite: batch studies and microscopic evidence for adsorption," Journal of Colloid and Interface Science, vol. 430, pp. 193-199, 2014.

[32] C. Hu, Y. Han, Y. Gao, Y. Zhang, and Z. Li, "Property investigation of calcium-silicate-hydrate (C-S-H) gel in cementitious composites," Materials Characterization, vol. 95, pp. 129-139, 2014. 
[33] K. Okano, M. Uemoto, J. Kagami et al., "Novel technique for phosphorus recovery from aqueous solutions using amorphous calcium silicate hydrates (A-CSHs)," Water Research, vol. 47, no. 7, pp. 2251-2259, 2013.

[34] Z. Deng, T. Wang, N. Zhang, and Z. Wang, "Gas holdup, bubble behavior and mass transfer in a $5 \mathrm{~m}$ high internal-loop airlift reactor with non-Newtonian fluid," Chemical Engineering Journal, vol. 160, no. 2, pp. 729-737, 2010.

[35] H. Huang, J. Yang, and D. Li, "Recovery and removal of ammonia-nitrogen and phosphate from swine wastewater by internal recycling of struvite chlorination product," Bioresource Technology, vol. 172, pp. 253-259, 2014. 

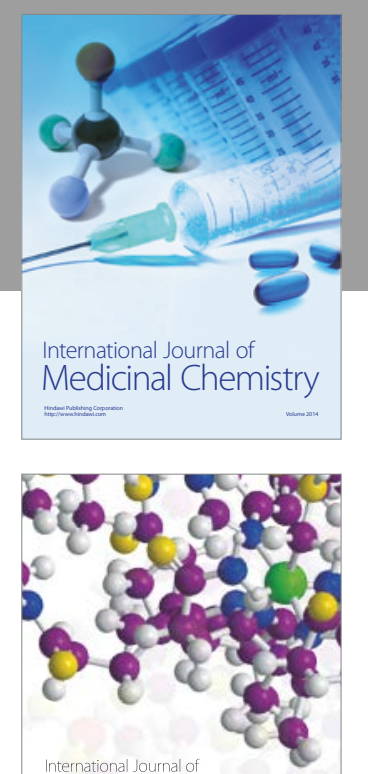

Carbohydrate Chemistry

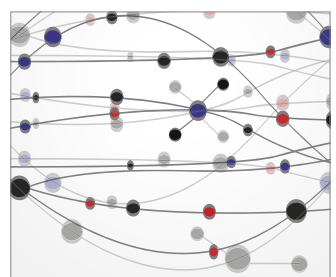

The Scientific World Journal
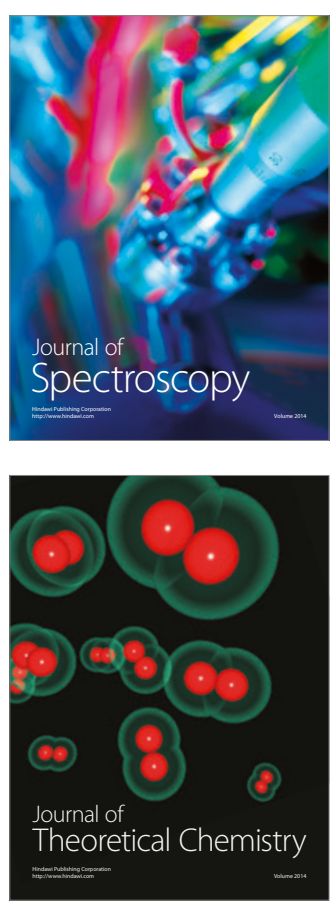
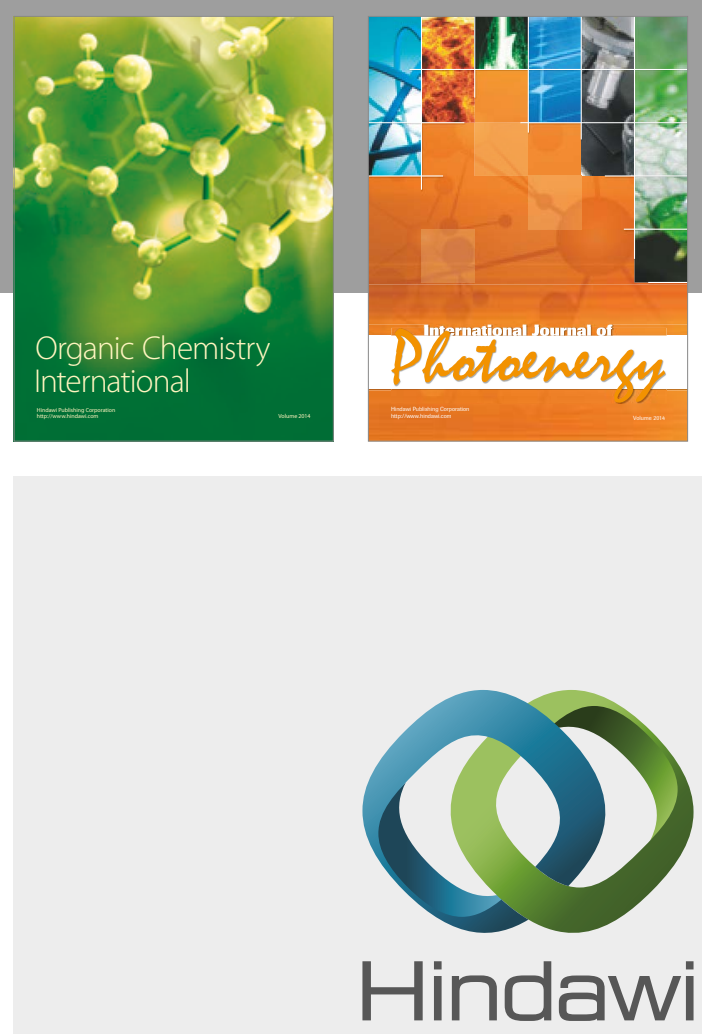

Submit your manuscripts at

https://www.hindawi.com

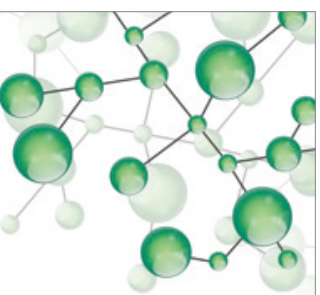

International Journal of

Inorganic Chemistry

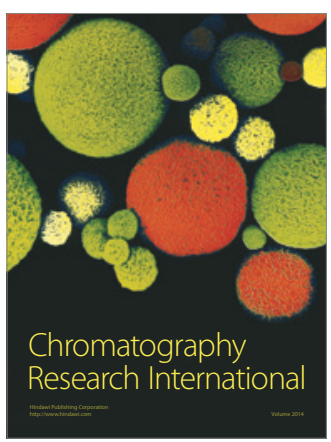

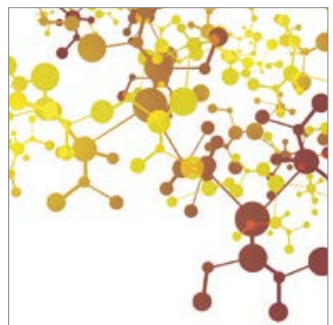

Applied Chemistry
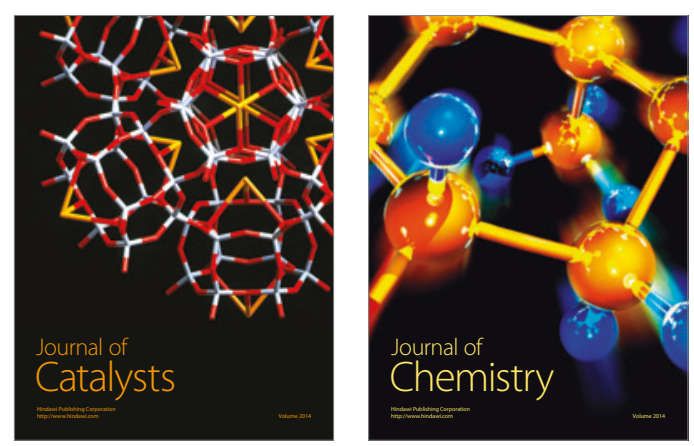
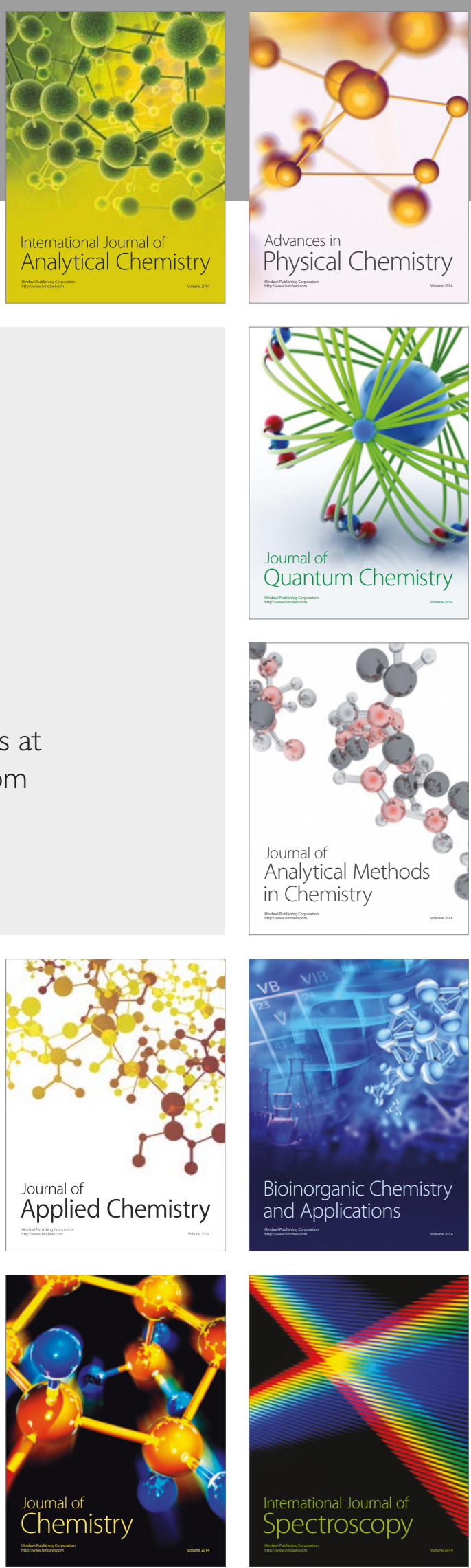